\title{
Preventive Analgesia and Novel Strategies for the Prevention of Chronic Post-Surgical Pain
}

\author{
Hance Clarke • Michael Poon • Aliza Weinrib • \\ Rita Katznelson · Kirsten Wentlandt • \\ Joel Katz
}

\begin{abstract}
Chronic post-surgical pain (CPSP) is a serious complication of major surgery that can impair a patient's quality of life. The development of CPSP is a complex process which involves biologic, psychosocial, and environmental mechanisms that have yet to be fully understood. Currently perioperative pharmacologic interventions aim to suppress and prevent sensitization with the aim of reducing pain and analgesic requirement in acute as well as long-term pain. Despite the detrimental effects of CPSP on patients, the body of literature focused on treatment strategies to reduce CPSP remains limited and continues to be understudied. This article reviews the main pharmacologic candidates for the treatment of CPSP, discusses the future of preventive analgesia, and considers novel strategies to help treat acute post-
\end{abstract}

H. Clarke · M. Poon · A. Weinrib · R. Katznelson · J. Katz

Pain Research Unit, Department of Anesthesia and Pain

Management, Toronto General Hospital, 200 Elizabeth Street,

Eaton North 3 EB 317, Toronto, ON M5G 2C4, Canada

e-mail: hance.clarke@utoronto.ca

H. Clarke · R. Katznelson · J. Katz

Department of Anesthesia, University of Toronto, Toronto, ON,

Canada

H. Clarke · A. Weinrib · R. Katznelson - K. Wentlandt · J. Katz Transitional Pain Service, Toronto General Hospital, Toronto, ON, Canada

\author{
K. Wentlandt \\ Department of Family and Community Medicine, University of \\ Toronto, Toronto, ON, Canada \\ K. Wentlandt \\ Palliative Care, Toronto General Hospital, Toronto, ON, Canada \\ J. Katz \\ Department of Psychology, York University, Toronto, ON, \\ Canada
}

operative pain and lessen the risk that it becomes chronic. In addition, this article highlights important areas of focus for clinical practice including: multimodal management of CPSP patients, psychological modifiers of the pain experience, and the development of a Transitional Pain Service specifically designed to manage patients at high risk of developing chronic post-surgical pain.

\section{Key Points}

The development of chronic post-surgical pain (CPSP) is a complex process which involves biologic, psychosocial, and environmental mechanisms.

Ketamine (an NMDA antagonist) appears to be the pharmacologic agent with the most consistent positive preventive analgesic results.

The variation in patient response to pharmacologic agents can be explained, in part, by genetic polymorphisms.

Understanding the heritability of CPSP will be useful when developing predictive algorithms to determine the preoperative risk for developing CPSP.

Psychological treatments delivered before and after surgery have the potential to influence the trajectory of CPSP from the earliest days, in combination with multimodal, preventive analgesia.

A novel multidisciplinary Transitional Pain Service involving early detection and management of patients at high risk for CPSP may modify the postsurgical pain trajectory and reduce the incidence of CPSP. 


\section{Introduction}

The development of chronic post-surgical pain (CPSP) is a serious complication of major surgery. While most surgical patients heal within months and return to their baseline functional status, certain surgical procedures and surgical candidates are at increased risk of developing CPSP and using opioid medications in the long-term $[1,2]$. Procedures associated with a high incidence of post-surgical pain include limb amputation (30-50\%), breast cancer surgery (20-30\%), thoracotomy (30-60\%) and heart bypass surgery $(30-50 \%)$ [3]. Currently, there is no universally agreed upon definition of CPSP. However, Macrae and Davies [1] define CPSP as pain that developed after a surgical procedure, of at least 2 months duration, and not the result of other causes or a pre-existing pain problem [4].

While CPSP is not exclusively neuropathic in origin, nerve injury and associated ectopic neural activity is implicated as an important inciting factor in many cases [5]. In addition, inflammatory and immune reactions, following axonal damage, result in the release of neurotransmitters that act both locally and in the spinal cord to produce hypersensitivity. In turn, central sensitization characterized by heightened dorsal horn neural activity and amplification of sensory flow may alter central nervous system function (CNS) and structure. Several proposed mechanisms for CPSP include: sensitization of peripheral nociceptors/associated primary afferents and the potential growth of aberrant neurons. These mechanisms lead to the clinical presentation of allodynia (pain following an innocuous stimulus) and hyperalgesia (an exaggerated response to painful stimulus), which are suggestive of neuropathic pain $[3,5]$. Despite the evidence in support of intraoperative nerve injury as a causal factor in the development of CPSP, not all patients with CPSP have documented nerve injury or neuropathic pain [6]. Moreover, many patients with objective signs of nerve injury do not develop CPSP [7]. The development of CPSP is a complex process which involves biologic, psychosocial, and environmental mechanisms that have yet to be fully understood [8].

Preventive analgesia is distinguished from pre-emptive analgesia in that the focus of treatment is less on the relative timing of the analgesic or anesthetic intervention and more on the mechanisms of action. Preventive analgesia aims to decrease the impact of the nociceptive barrage associated with noxious pre-operative, intra-operative, and/or post-operative events/stimuli [9]. Currently perioperative pharmacologic interventions aim to suppress and prevent sensitization with the goal of reducing pain and analgesic requirements in acute as well as long-term pain. For the purposes of this review, a preventive analgesic effect is demonstrated when post-operative pain and/or analgesic use are reduced beyond the clinical duration of action of the target agent which we have defined as 5.5 half-lives of the target agent. This requirement ensures that the observed effects are not analgesic effects [10].

The most common medication classes that have been investigated as adjunctive medications to opioid-based analgesia in the perioperative setting include: anticonvulsants, non-steroidal anti-inflammatories (NSAIDs), NMDA receptor antagonists, local anesthetics, and antidepressants/ anxiolytics. A recent systematic review on the subject related to this concept was published by Chaparro et al. [11], examining pharmacologic interventions for CPSP. As there remains considerable uncertainty with respect to the pharmacologic strategies best suited to reduce the development of CPSP, and in fact whether this is even possible, this review seeks to (1) update the Chaparro et al. review to obtain the most recent published studies on the topic, (2) discuss the efficacy of relevant drugs currently used for CPSP, and (3) introduce novel clinical strategies aimed at understanding the mechanisms underlying the development of CPSP. Not only are pharmacologic interventions important to mitigate development of CPSP, psychological management of patients with co-morbid psychological vulnerabilities are needed in order to help improve the recovery trajectories of complex perioperative pain patients.

\section{Literature Update}

A systematic review of pharmacologic management of CPSP was performed by Chaparro et al. [11]. In the present narrative review, the Chaparro et al. search strategy was implemented for the past year (January 2013-July 2014) in order to provide an up-to-date comprehensive view of current literature.

\subsection{Primary Outcome}

Studies reporting one or both of the following outcomes were included for review in the present paper. Patient-reported measures of pain 2 months or more following a surgical procedure were included.

1. Proportion of participants reporting any pain at the anatomical site of the procedure or pain around the scar.

2. Severity of pain reported by participants at the anatomical site 2 months or more after surgery. 


\subsection{Inclusion Criteria for Studies}

Double-blind, randomized trials of one or more drugs administered before, during, or after surgery; pain measured using a validated pain assessment instrument 2 months or more after surgery; participants of both genders; 18 years of age or older; undergoing a planned surgical procedure.

\subsection{Search Strategy}

An update of the Chaparro et al. [11] review of pharmacotherapy for chronic post-surgical pain was conducted from 2013 to 14 July 2014 using the same search strategy. The literature search was conducted in MEDLINE, EMBASE, and the Cochrane Central Register of Controlled Trials on the OvidSP platform to identify randomized controlled trial results for chronic post-surgical pain using main pharmacologic candidates to date. This included anticonvulsants, ketamine, local anesthetics, antidepressants, anxiolytic agents, and NSAIDs.

\section{Search Results}

The systematic search, conducted in August 2014 using the Chaparro et al. search terms, identified 364 citations. The results of the search were initially screened by two of the review authors (MP \& HC) based on the title of publication and abstract contents. Screening of citation abstracts yielded 15 records which were reviewed in full text. After full-text review, nine studies fulfilled the inclusion criteria and were included in the present review (Fig. 1). Five of the nine studies examined local anesthetics in several surgical populations [12-16], one study compared a tumor necrosis factor-alpha (TNF- $\alpha$ ) inhibitor (i.e., enteracept) for inguinal hernia compared to placebo [17], another study compared pregabalin versus placebo in a cardiac surgery model [18], and another study examined minocycline (a broad-spectrum cycline antibiotic which has been shown to block microglial activation) compared to placebo following lumbar discectomy [19]. These eight trials were negative and did not demonstrate a preventive analgesic effect [1219]. Only one of the nine studies identified in our updated literature search found a preventive effect on the incidence of CPSP [20]. Patients who underwent total abdominal hysterectomy and were induced and maintained solely with propofol were found to have a significantly lower incidence of CPSP (7/40 patients) compared to those who were induced with propofol and then maintained with sevoflurane (20/40 patients) for their general anesthetic [20]. Although the mean pain scores were significantly lower in the propofol group $(0.78 \pm 0.55)$ compared to the sevoflurane

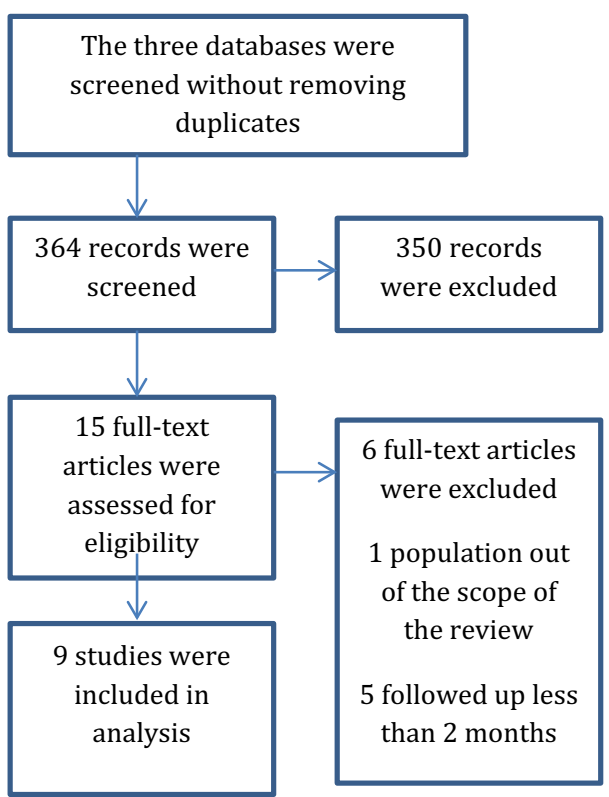

Fig. 1 Chaparro update 2013-2014 study flow diagram

group $(2.23 \pm 0.73, p=0.01)$, scores for both groups were in the mild range.

The results of the systematic review update are summarized in Table 1.

\section{Pharmacologic Management for the Prevention of Chronic Post-Surgical Pain (CPSP)}

\subsection{Anticonvulsants}

\subsubsection{Gabapentin}

The antinociceptive effects of gabapentin result, in part, from its high affinity for the $\alpha_{2} \delta$ subunit on pre-synaptic voltage-gated calcium channels, which are often upregulated following nerve injury. This serves to inhibit calcium influx and the release of excitatory neurotransmitters, which elicits the sensation of pain [21]. One recent meta-analysis supported the view that perioperative administration of gabapentin was effective in reducing the incidence of CPSP [22]. In contrast, in a more recent systematic review by Chaparro et al. [11] on the use of gabapentin for 3-month postoperative pain, none of the included five studies demonstrated a significant difference over placebo [11]. A total of 280 patients were analyzed, of whom 71/133 patients receiving gabapentin and 79/147 patients receiving placebo went on to develop CPSP [11]. A similar non-significant result when comparing gabapentin and placebo was found for 6-month postoperative pain. However, at the 6-month time-point, it is important to 


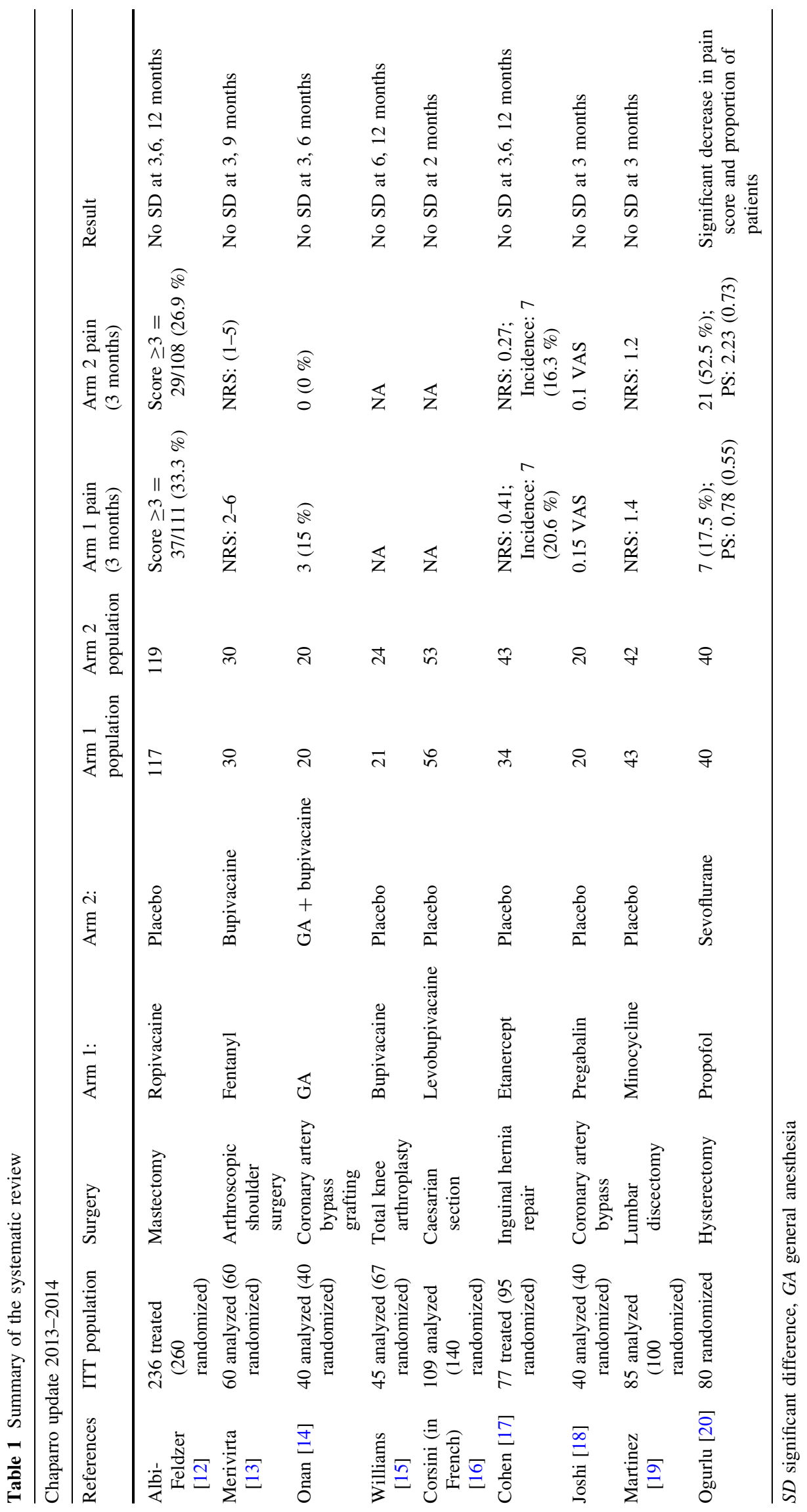


recognize that only two studies were included in the analysis [23, 24]. In two studies that were excluded from Sen et al. [25, 26], postoperative pain scores were significantly lower in the gabapentin group compared with both the ketamine and control groups at 3- and 6-month follow-up following hysterectomy and inguinal herniorrhaphy, respectively. In the remaining excluded gabapentin trial, no significant difference was found between gabapentin and placebo for pain characteristics at 6 months [27]. Although the conclusion from Chaparro et al. was that that gabapentin does not reduce the incidence of CPSP, the small sample size of the studies limits these interpretations, especially when considering that these results derive from heterogeneous surgical procedures. Brogly et al. [28] found that $1200 \mathrm{mg}$ of gabapentin administered before surgery reduced chronic but not early acute postoperative pain following superficial cervical plexus block [28]. The lack of standardized gabapentin doses between studies may have contributed to inconsistencies in the drug's efficacy at reducing the incidence and severity of CPSP. It has been well documented that the absorption profile of gabapentin in humans is inconsistent due to the active and saturable $\alpha$ amino acid transport system [29]. Thus, the bioavailability of any given dose varies from 35-90\% [29]. Without plasma samples, one cannot confirm therapeutic drug concentrations of gabapentin, as such it is possible that the higher doses of gabapentin may enable therapeutic plasma levels needed to demonstrate preventive effects with respect to the reduction of CPSP. Another possibility for the lack of an effect on CPSP is the heterogeneity of the surgical populations studied to date, perhaps if focused on procedures that are associated with significant neuropathic pain (i.e., spine, joint, thoracotomy, and amputations) the preventive benefits of gabapentinoid perioperative administration may become evident. However, in a recent study outside of the 1-year literature update, gabapentin failed to reduce the incidence or intensity of CPSP when compared to placebo 3 months after total knee arthoplasty [30]. Recently, the available literature indicated that perioperative gabapentin was effective in preventing CPSP [22]; however, Chaparro et al. [11] suggest equivalence to placebo in their review.

\subsubsection{Pregabalin}

Pregabalin is a structural analog of $\gamma$-aminobutyric acid. Similar to gabapentin, pregabalin inhibits $\mathrm{Ca}^{2+}$ currents by binding the $\alpha_{2} \delta_{1}$ subunit on pre-synaptic voltage-gated calcium channels [31]. In Chaparro et al.'s [11] systematic review, five pregabalin trials with long-term pain outcomes were previously identified, of which two studies demonstrated a significant benefit of pregabalin as compared to placebo $[32,33]$. While the results between the studies were quite heterogeneous at 3-month follow-up, an overall significant effect of pregabalin was found [11]. Forty-eight of 217 patients developed CPSP in the pregabalin group compared to 70 of 222 patients in the placebo group. The literature update identified one study that did not find a significant difference between patients that received $150 \mathrm{mg}$ of pregabalin $2 \mathrm{~h}$ prior to induction of anesthesia and $75 \mathrm{mg}$ twice daily for two postoperative days compared to placebo in 40 patients undergoing coronary artery bypass surgery 3 months following surgery [18]. Buvanendran et al. [33] did find a significant reduction in the incidence of CPSP 6 months following total knee arthroplasty in patients who were randomized to receive a $300-\mathrm{mg}$ preoperative dose of pregabalin followed by a 14-day twice-a-day (BID) regimen of pregabalin $(50-150 \mathrm{mg})$ or placebo [33]. The heterogeneity of dosing regimens studied to date is problematic with respect to comparing long-term outcomes. Overall, despite the heterogeneity between studies, available literature favors the perioperative use of pregabalin to prevent CPSP; however, when unpublished data are taken into consideration [34] and in light of a new publication which is outside of this review [35], this conclusion needs to be reassessed.

\subsection{Non-Steroidal Anti-Inflammatory Drugs (NSAIDs)}

The analgesic effects of NSAIDs have been attributed to their anti-inflammatory properties with respect to inhibiting the synthesis of prostaglandins [36]. The identification of two COX isoforms, COX-1 and COX-2, which catalyze the first two reactions in the prostaglandin pathway enabled efforts to characterize the relative contribution of each isoform to inflammation and pain [37]. The COX-2 isoform appears to be the enzyme responsible for inflammation and pain. COX-2 inhibitors, such asparecoxib, reduce secondary hyperalgesia and appear to reduce central sensitization in humans $[38,39]$. Celecoxib (the only COX-2 inhibitor approved in the North American marketplace) has demonstrated a reduction in postoperative pain, a reduced need for postoperative opioid analgesia [40], and did not inhibit bone healing following arthroplasty surgery [41]. There is no meta-analysis of NSAIDs for CPSP currently in the literature. Clinical trials are heterogeneous and differ in type of drug used, follow-up time-point, and pain outcomes. Three prospective, randomized perioperative trials have reported results of NSAIDs for CPSP [42-44], none of which have demonstrated a significant impact of NSAIDs (in isolation) with respect to a reduction in incidence or severity of CPSP [42-44]. No additional trials were identified in the 1-year literature update performed in this review. 


\subsection{N-Methyl-D-Aspartate (NMDA) Receptor Antagonists}

\subsubsection{Ketamine}

The NMDA receptor plays a critical role in both the induction and maintenance of central sensitization and pathologic pain [45]. Ketamine (a non-competitive NMDA receptor antagonist) is believed to reduce pain and analgesic consumption through the prevention of NMDA-mediated sensitization of spinal cord dorsal horn neurons [46]. Fourteen trials comparing ketamine to placebo for CPSP have been previously identified [11], of which 12 were used in the Chaparro et al. [11] systematic review [4754].The majority of trials identified used pre-incisional loading doses of ketamine ranging from 0.15 to $1 \mathrm{mg} / \mathrm{kg}$, plus an intraoperative infusion. A total cumulative dose of less than $1 \mathrm{mg} / \mathrm{kg}$ was reached in 6/14 studies, $4 / 14$ studies administered a cumulative dose of ketamine between 1 and $2 \mathrm{mg} / \mathrm{kg}$, and $4 / 14$ administered a dose greater than $2 \mathrm{mg} /$ $\mathrm{kg}$ [11]. While ketamine showed a non-significant effect for the reduction of CPSP 3 months after surgery, a subgroup analysis of trials with patients receiving longer than $24 \mathrm{~h}$ of ketamine treatment demonstrated ketamine's superiority over placebo [11]. A more definitive result was found for ketamine 6 months following surgery, with an overall significant decrease in the incidence of CPSP. Six months following surgery, studies in which patients received ketamine for less than $24 \mathrm{~h}$ demonstrated a reduction in the incidence of CPSP compared to studies in which patients received ketamine for more than $24 \mathrm{~h}$. A total of 55 of 285 patients on ketamine developed CPSP at 6 months, compared to 79 of 231 patients who received a placebo [11]. Two trials that examined the effects of ketamine administration were excluded from the Chaparro et al. systematic review as they did not report the outcomes of interest. In the first study, postoperative pain scores were significantly lower in patients treated with gabapentin as compared to patients who received ketamine or placebo. In this trial, a $0.3 \mathrm{mg} / \mathrm{kg}$ intravenous (IV) bolus and $0.05 \mathrm{mg} /$ $\mathrm{kg}$ per hour infusion of ketamine was not found to be significantly different to placebo [25]. In a study of 120 patients, Dullenkopf [55] did not find a difference in pain scores at 3 months between patients treated with placebo and either $0.15 \mathrm{mg} / \mathrm{kg}$ ketamine IV or $0.5 \mathrm{mg} / \mathrm{kg}$ ketamine IV given pre-incision. While the use of ketamine to reduce CPSP is empirically promising, the results still remain controversial due to the wide variability in clinical settings, ketamine regimens, and reported outcomes. In addition, some of the positive results have been obtained using clinical anesthesia regimens which are not accepted as standard treatments $[55,56]$. Since Chaparro et al.'s systematic review no ketamine trials were identified which examined CPSP outcomes. Overall, the available literature supports the perioperative use of ketamine to prevent CPSP.

\subsubsection{Other NMDA Receptor Antagonists}

Other NMDA receptor antagonists have not been studied as extensively as ketamine. In one study of 19 patients, memantine was found to significantly reduce the incidence and intensity of phantom limb pain in patients undergoing traumatic amputations when used in combination with a regional block [57]. Another study on the effects of amantadine for CPSP found that all patients treated with amantadine developed CPSP (9/9) compared to $75 \%$ of patients who received a placebo (6/8) [58]. Further study of these agents may be warranted. No studies were identified with our literature update in the past year which examined memantine or amantadine for the reduction of CPSP. The current body of literature available is insufficient to determine efficacy.

\subsection{Local Anesthetics}

Local anesthesia is an important adjunct used to decrease pain, opioid consumption, and opioid-related adverse effects [59]. For CPSP, local anesthetics may have clinical utility through early interruption of sensory information to the dorsal horn of the spinal cord [60]. The analgesic action from local anesthetics is based on the suppression of abnormal sodium channels that exist along injured neurons via cellular mechanisms such as the inhibition of $G$ proteincoupled receptors and NMDA receptors [61, 62]. Independent studies have sought to determine the effect of several different local anesthetics on CPSP. Patients who received intravenous lidocaine during breast cancer surgery had a lower incidence of CPSP 3 months following surgery [63]. Patients randomized to IV lidocaine were found to have significantly lower pain scores, with fewer patients developing CPSP (2/17 patients) or secondary hyperalgesia compared to patients randomized into the placebo group (9/ $19)$ at 3 months. Fassoulaki et al. [60] evaluated the use of mexiletine in a pair of clinical trials. In a randomized, placebo-controlled trial, the incidence of CPSP and pain scores did not differ between study arms. Similarly, in their second trial comparing mexiletine to gabapentin and placebo, no significant difference was found between trial groups [64]. Within the past year, an additional five studies have been published evaluating the use of local anesthetics for CPSP [12-16]. No significant difference in incidence or pain score was found for any of the agents 2 months or more following the surgery. While there is some evidence in support of the use of local anesthetics, the agents used are heterogeneous and the sample sizes quite small. Further 
investigation is needed in order to draw more meaningful conclusions. To that end, different routes of delivery for local anesthestics (beyond PO, neuraxial, and IV), such as medial open transversus abdominis plane (MOTAP) catheters which deliver local anesthetic into the abdominal cavity to anesthetize the neural plexus supplying the anterior abdominal wall cephalad to the umbilicus (dermatomes T6-10), are currently being investigated in the perioperative setting following liver resection and other major surgical interventions to study secondary effects such as the development of CPSP [65]. To date, the available literature suggests that local anesthetics as a class are equivocal to placebo for CPSP. However, there is limited evidence that favors the use IV lidocaine for CPSP.

\subsection{Selective Norepinephrine and Serotonin Re-Uptake Inhibitors (SNRIs)}

Venlafaxine hydrochloride is an antidepressant medication that inhibits the neuronal reuptake of serotonin and norepinephrine. However, it has a better safety profile as it does not bind to cholinergic, histamine, or $\alpha_{1}$-adrenergic binding sites [66]. In a study comparing venlafaxine to gabapentin and placebo, venlafaxine was found to significantly reduce the incidence of CPSP at 6 months in women having breast surgery [27]. The literature regarding SNRI use for the prevention of chronic post-surgical pain is limited; however, given that the SNRI medication (duloxetine) is a firstline medication for the treatment of chronic neuropathic pain [67], its role in the prevention of chronic post-surgical pain should be further investigated. Duloxetine has been shown to be efficacious for the treatment of diabetic peripheral neuropathy and fibromyalgia [68-71]. Appropriately powered trials are necessary to examine the efficacy of the SNRIs in CPSP prevention, including dose-response studies and head-to-head comparisons with other drugs, such as pregabalin. In the past year, no new studies examined the role of the SNRIs for the reduction of CPSP. The current literature regarding SNRIs is insufficient to determine efficacy with respect to the prevention of CPSP and further studies are needed.

\subsection{Multimodal Analgesia}

The concept of multimodal analgesia for treating perioperative pain has become widely accepted and standard multimodal analgesic regimens tailored to specific surgical procedures have been adopted by many institutions [18] Utilizing different classes of medication to target different peripheral and CNS mechanisms to reduce acute pain, reducing opioid analgesic requirements postoperatively and improving opioid related side effects has been shown to be successful in clinical trials $[60,64]$. However, RCTs that examine specific surgery-related multimodal regimens versus placebo and follow patients for preventive analgesic effects (i.e., 3 months or greater following surgery) are limited. We identified two trials comparing multimodal analgesic regimens to placebo in the published literature, both of which demonstrated positive preventive effects at 3 months [72] and the other at 1 year [73] following surgery. Multimodal pain regimens that include gabapentin, NSAIDs, acetaminophen, and regional anesthesia were effective in controlling perioperative pain and decreasing perioperative opioid use following arthroplasty surgery $[74,75]$. The literature has highlighted the need for studies focused on multimodal analgesic protocols for colorectal [76], thoracic, amputation, mastectomy [77], orthopaedic [37], and liver resection surgeries [65]. In these trials, the outcome of interest should not only focus on reducing acute postoperative pain and opioid consumption (due to the immediate drug effect), but also on ascertaining whether preventive effects that reduce the incidence and severity of CPSP are demonstrated.

\section{Genetics of CPSP}

There is a large variability with respect to the development of CPSP amongst patients undergoing the same surgery. This individual variability suggests that there are several factors which contribute to the development of CPSP (both genetic and environmental). Recent reviews in pain genetics estimate that chronic pain heritability ranges from 30 to $70 \%$ [78]. Presently it is not possible to ascertain the extent to which individual genetic factors contribute to a person's propensity to develop chronic postsurgical pain or their response to therapy [79].

A number of candidate genes have been identified for chronic pain, including a few for post-surgical and posttraumatic pain. These include genes encoding for ion channels, neurotransmitter enzymes, receptors, transporters, transcription factors, and hormone receptors that increase the risk for pain chronicity [79]. While there are numerous studies that are ongoing, variations of three genes mapped in rodent models have been associated with chronic pain in humans. These candidate pain genes are KCNSI (encoding the s1 subunit of a voltage-dependent potassium channel), CACNG2 (encoding the gamma subunit 2 of a voltage-gated calcium channel), and the P2RX7 gene (encoding the $\mathrm{P}_{2} \mathrm{X}_{7}$ purinergic receptor, an ionotrophic adenosine-triphosphate-gated receptor that operates purinergic synapses in the CNS) [80-82]. In addition to polymorphisms that affect the development of chronic pain, polymorphisms can affect a patients' response to pharmacologic agents. Specificpolymorphisms that influence the metabolism of medications have been 
identified. The identified polymorphisms affect the cytochrome P450 enzymatic pathway [83]. Therapeutic response to medications can be guided with known genetic information and patients can be classified as a normal, intermediate, poor, rapid, or ultrarapid metabolizer. For opioid metabolism, the relationship of pain and polymorphisms of cytochrome P450 enzymes (CYP2D6, CYP2B6, and CYP3A4) are well documented [83]. In a study by Yang et al. [84], $71 \%$ of postoperative patients with acute severe pain had the CYP2D6 poor metabolizer genotype. In a separate analysis, ultrametabolizers for CYP2D6 also demonstrated higher efficiency in synthesizing endogenous morphine compared to other CYP metabolizer groups and required significantly less morphine in the postoperative period [85].

Presently, it is not possible to predict which individuals may be susceptible to the development of CPSP. However, it is important to recognize that gene polymorphisms that exist in a patient population may end up determining different responses to treatment. The ultimate goal of pain genetics is to enable clinicians to predict preoperatively who is at risk of developing CPSP and translate this understanding into pharmacologic and surgical decisionmaking. Improved knowledge could contribute to personalized preventive pain treatments and allow management to move away from the current symptom-based approach [79].

\section{Development of a Transitional Pain Service}

The call for the implementation of clinical programs and research into risk factors associated with the transition of acute to chronic post-surgical pain has been longstanding [86-88]. However, equally problematic but less often discussed is the issue of prolonged opioid use associated with CPSP. Given that one in 33 previously opioid-naïve patients who undergo major surgery continue to refill their opioid prescription up to 6 months following surgery [2], the time has come to focus on the antecedent predictors of persistent opioid use and the development of post-surgical pain. Furthermore, there is a vulnerable population of complex pain patients (often excluded from randomized controlled trials) who present with pre-existing pain syndromes and who use significant amounts of opioid analgesics pre- and perioperatively. These patients are often managed quite poorly and may leave the hospital on more analgesic medications than when they were triaged presurgically $[89,90]$. It is important to prospectively determine which patients should be aggressively treated within the perioperative time period in the hopes of reducing the alarming percentage of patients $(25 \%)$ referred to tertiary pain care centers presenting with a CPSP condition [4].
We have developed a Transitional Pain Service (TPS) to target patients at high risk for the development of acute and chronic post-surgical pain, as well as the development of persistent opioid use following surgery. These include but are not limited to patients with (1) a current/previous history of chronic pain; (2) previous/current drug misuse or abuse; (3) patients with previous/current psychological comorbidities; (4) current/previous opioid therapy for pain; (5) patients on methadone or buprenorphine; (6) patients followed by our Acute Pain Service for an extended period of time for poorly managed pain; (7) patients consuming excessive amounts of postoperative opioids (e.g., $>90 \mathrm{mg} /$ day); and (8) any patient referred to the TPS by an attending surgeon at our facility. Once a patient has been identified as requiring the services of the TPS, a nurse practitioner commences a program of enhanced teaching with the patient while in hospital. Communication with primary care is initiated while in hospital. Once a patient has been deemed medically fit for discharge, patients are sent home with a TPS clinic visit scheduled within days of discharge to see one of the TPS attending physicians (while receiving follow-up calls from the TPS team coordinator until the scheduled appointment).

At the initial visit to the outpatient TPS clinic after surgery, a detailed pain treatment plan including an opioidweaning strategy is discussed with each patient (and family members if present). Patients are assessed for opioid addiction risk and an opioid agreement contract is signed if necessary. Patients are assessed once every 2 weeks and their opioid medications and other analgesics are adjusted until they are at a safe level, their pain is under control, and their daily function approaches their pre-surgical level (or better). The aim is to transition patients back to their primary-care physicians within 6 weeks to 3 months of hospital discharge (i.e., after 3-6 visits).

For these patients, we are currently combining perioperative psychological treatments (e.g., Acceptance and Commitment Therapy), as well as holistic alternative medicine treatments (e.g., acupuncture), and traditional pharmacologic management. In preliminary analyses, patients report decreased pain intensity over time while being treated by TPS. Pain trajectory data from the first 4 months of our service show lower numeric pain rating (NRS) scores (NRS pain at discharge $5.1 \pm 2.7$, and at 2 months $2.7 \pm 2.9$ ) when compared to a non-TPS thoracic surgery cohort of patients (NRS pain at discharge $7.7 \pm 1.6$, at 3 months $4.8 \pm 1.5$ ) followed prospectively in a TGH Pain Research Unit study (Fig. 2). The data also indicates better hospital pain management $(p<0.05)$ and a faster trajectory to mild pain $(\mathrm{NRS}<4$ ) with TPS involvement.

Cousins et al. [91] have identified the economic burden of acute postoperative pain that progresses to a chronic pain syndrome in a 30 -year-old individual is as much as 


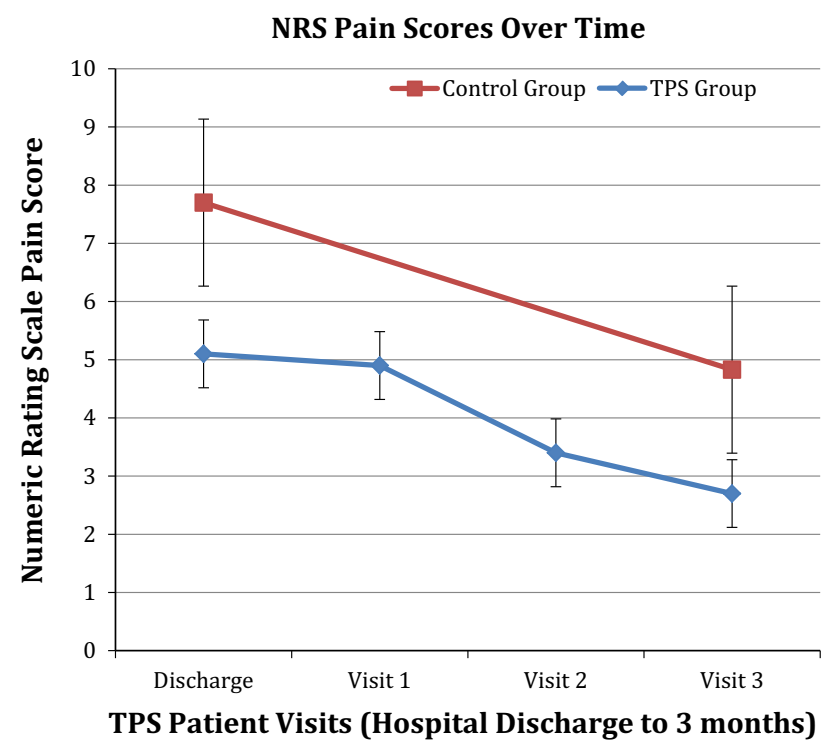

Fig. 2 Comparison of numerical pain rating scale (NRS) scores for patients followed by a Transitional Pain Service (TPS) compared to a thoracic surgery cohort control group

US\$1 million over the course of their lifetime [91]. The TPS has been developed and designed to identify complex perioperative pain patients at risk for the development of CPSP, institute early interventional practices, reduce the wait time for referral back to chronic pain specialists, and save the health-care system significant direct and indirect costs.

\subsection{Psychological Predictors and Means of Intervening}

Elsewhere we have argued that the true power of preventive, multimodal treatment to impact the trajectory of postsurgical pain can only be realized by the integration of psychological interventions into the patients' pre- and postsurgical care [8]. Pain after surgery-in the aftermath of incision, intra-operative tissue damage and the ensuing inflammatory cascade-is subject to a host of psychosocial influences, including those related to mood and attention [92]. Psychosocial risk factors of particular concern with respect to CPSP include pre-surgical depression, anxiety, surgical fear [93], and emotional numbing [94]. Pain catastrophizing (rumination, magnification, and helplessness) after surgery may confer added risk of CPSP, although findings have been mixed and may be surgeryspecific [8]. Innovative research from our group has identified subgroups of patients with distinct post-surgical pain trajectories. Both pre-operative pain and anxiety are risk factors for continued pain at 12 months after TKA [95] and we have provided evidence that pain catastrophizing predicts chronic post-surgical pain following TKA in a recent systematic review [96]. Over the long term, persistent pain increases vulnerability to distress, which potentiates pain perception, and can lead to a vicious cycle in which pain and distress amplify one another [94].

Psychological treatment (most frequently cognitive-behavioral therapy) is a well established and empirically supported component of multidisciplinary pain programs that has been shown to interrupt this kind of vicious cycle and lead to improved patient outcomes [97]. Patients who have participated in psychological interventions for chronic pain (including Cognitive Behavioral Therapy [98] and Acceptance and Commitment Therapy [99, 100]) report less pain, pain-related disability, and mood disturbance. Despite the effectiveness of these programs in terms of the patient's quality of life, chronic pain patients often have difficulty accessing them until pain is well established and all other medical options have been exhausted. In contrast, for most surgeries, the nature of the injury and time of onset of pain are known in advance and patients are accessible through the hospital system [8]. Surgery therefore offers a unique opportunity to reach patients early-even before surgery-to influence psychological variables such as pre-surgical anxiety. Early evidence indicates this approach is promising in terms of outcomes in the acute postsurgical time period [101, 102]; the impact of psychological intervention on the prevention of persistent pain is yet to be demonstrated. Psychological treatments delivered at the pre-surgical and acute post-surgical time points have the potential to influence the trajectory of CPSP from the earliest days, in combination with multimodal, preventive analgesia. There is a growing call for such interventions and they are in the early stages of being developed and empirically tested $[93,103]$.

\section{Future Directions}

The heterogeneity with respect to the timing of interventions, the time points studied, and the outcomes assessed limit meaningful comparison of CPSP trials to date .There were two negative trails identified in this review which focused on minocycline [19] and entanercept [17] as possible agents that may play a role in modifying the transition from an acute pain to chronic pain. The role of tumor necrosis factor-alpha (TNF- $\alpha$ ) inhibitors and medications implicated in the blocking of microglial activation deserve further study. Future trials should focus on the acute and subacute postoperative period and early pharmacologic and non-pharmacologic interventions that may modify the pain trajectory and reduce the incidence of CPSP. Standardization of methodology and reporting of CPSP studies is required to elucidate future optimal management of patients experiencing CPSP. It is also important to recognize that not all pain can be eliminated. Consequently, goal- 
related therapies aimed at an improvement in quality of life and function are potentially more appropriate outcomes than a reduction in numeric pain rating scales 3 or 6 months following major surgery.

\section{Conclusions}

In the past year, the updated literature search identified only one randomized trial which demonstrated a significant reduction in CPSP. The focus on treatment strategies aimed at the reduction of CPSP continues to be a field in which much more research is needed. The heterogeneity of dosing regimens complicates the ability to draw meaningful conclusions with respect to the preventive effects of each pharmacologic agent. Future studies should focus on procedures that elicit pronociceptive (acute hyperalgesia) mechanisms (e.g., joint arthroplasty, thoracotomy) because studying surgeries that have a higher incidence/severity of CPSP would increase the likelihood of revealing preventive effects. Using the IASP recommendations for the assessment of core measures and domains in clinical trials which includes relevant psychological, emotional, and physical variables in addition to those routinely assessed (i.e., pain and analgesic use), may highlight predictors which increase the risk for developing chronic post-surgical pain [104]. Moving forward, effects of multimodal analgesic regimens should be studied beyond 2 months following surgery in order to determine whether multimodal pharmacotherapy is effective in reducing the development of CPSP. The implementation of a Transitional Pain Service will enable early referral for interventional pain procedures, psychoeducation, and targeted mental health interventions for patients identified as at high risk for CPSP. These services tailored specifically to the individual surgical patient are aimed at improved in-hospital acute pain control, lower post-discharge pain scores, effective weaning of opioid medications, improved quality of life, and reduced pain disability, as well as a significantly lower risk of CPSP.

Acknowledgments $\mathrm{HC}$ is supported by a Merit Award (Department of Anaesthesia, University of Toronto) and the STAGE Training Program in Genetic Epidemiology (Canadian Institutes of Health Research, CIHR) and a grant by the Physicians Services Incorporated Foundation. JK is supported by a Canada Research Chair in Health Psychology. The authors of this manuscript have no conflicts of interest to declare.

\section{References}

1. Searle R, Simpson K. Chronic post-surgical pain. Contin Educ Anaesth Crit Care Pain. 2010;10(1):12-4.
2. Clarke H, Soneji N, Ko DT, Yun L, Wijeysundera DN. Rates and risk factors for prolonged opioid use after major surgery: population based cohort study. BMJ. 2014;11(348):g1251.

3. Perkins FM, Kehlet H. Chronic pain as an outcome of surgery. A review of predictive factors. Anesthesiology. 2000;93(4): 1123-33.

4. Macrae WA. Chronic post-surgical pain: 10 years on. $\mathrm{Br} \mathrm{J}$ Anaesth. 2008;101(1):77-86.

5. Kehlet H, Jensen TS, Woolf CJ. Persistent postsurgical pain: risk factors and prevention. Lancet. 2006;367(9522):1618-25.

6. Steegers MA, Snik DM, Verhagen AF, van der Drift MA, Wilder-Smith $\mathrm{OH}$. Only half of the chronic pain after thoracic surgery shows a neuropathic component. J Pain. 2008;9(10):955-61.

7. Ivens D, Hoe AL, Podd TJ, Hamilton CR, Taylor I, Royle GT. Assessment of morbidity from complete axillary dissection. $\mathrm{Br} \mathrm{J}$ Cancer. 1992;66(1):136-8.

8. Katz J, Seltzer Z. Transition from acute to chronic postsurgical pain: risk factors and protective factors. Expert Rev Neurother. 2009;9(5):723-44.

9. Pogatzki-Zahn EM, Zahn PK. From preemptive to preventive analgesia. Curr Opin Anaesthesiol. 2006;19(5):551-5.

10. Katz J, Clarke H, Seltzer Z. Review article: preventive analgesia: quo vadimus? Anesth Analg. 2011;113(5):1242-53.

11. Chaparro LE, Smith SA, Moore RA, Wiffen PJ, Gilron I. Pharmacotherapy for the prevention of chronic pain after surgery in adults. Cochrane Database Syst Rev. 2013;7:CD008307.

12. Albi-Feldzer A, Mouret-Fourme EE, Hamouda S, Motamed C, Dubois PY, Jouanneau L, et al. A double-blind randomized trial of wound and intercostal space infiltration with ropivacaine during breast cancer surgery: effects on chronic postoperative pain. Anesthesiology. 2013;118(2):318-26.

13. Merivirta R, Aarimaa V, Aantaa R, Koivisto M, Leino K, Liukas A, et al. Postoperative fentanyl patch versus subacromial bupivacaine infusion in arthroscopic shoulder surgery. Arthroscopy. 2013;29(7):1129-34.

14. Onan B, Onan IS, Kilickan L, Sanisoglu I. Effects of epidural anesthesia on acute and chronic pain after coronary artery bypass grafting. J Card Surg. 2013;28(3):248-53.

15. Williams D, Petruccelli D, Paul J, Piccirillo L, Winemaker M, de Beer J. Continuous infusion of bupivacaine following total knee arthroplasty: a randomized control trial pilot study. J Arthroplasty. 2013;28(3):479-84.

16. Corsini T, Cuvillon P, Forgeot A, Chapelle C, Seffert P, Chauleur C. Single-dose intraincisional levobupivacaine infiltration in caesarean postoperative analgesia: a placebo-controlled double-blind randomized trial. Ann Fr Anesth Reanim. 2013;32(1):25-30.

17. Cohen SP, Galvagno SM, Plunkett A, Harris D, Kurihara C, Turabi A, et al. A multicenter, randomized, controlled study evaluating preventive etanercept on postoperative pain after inguinal hernia repair. Anesth Analg. 2013;116(2):455-62.

18. Joshi SS, Jagadeesh AM. Efficacy of perioperative pregabalin in acute and chronic post-operative pain after off-pump coronary artery bypass surgery: a randomized, double-blind placebo controlled trial. Ann Card Anaesth. 2013;16(3):180-5.

19. Martinez V, Szekely B, Lemarie J, Martin F, Gentili M, Ben Ammar S, et al. The efficacy of a glial inhibitor, minocycline, for preventing persistent pain after lumbar discectomy: a randomized, double-blind, controlled study. Pain. 2013;154(8):1197-203.

20. Ogurlu M, Sari S, Kucuk M, Bakis M, Ugur B, Eshraghi YE, et al. Comparison of the effect of propofol and sevoflurane anaesthesia on acute and chronic postoperative pain after hysterectomy. Anaesth Intensive Care. 2014;42(3):365-70. 
21. Luo ZD, Calcutt NA, Higuera ES, Valder CR, Song YH, Svensson CI, et al. Injury type-specific calcium channel alpha 2 delta-1 subunit up-regulation in rat neuropathic pain models correlates with antiallodynic effects of gabapentin. J Pharmacol Exp Ther. 2002;303(3):1199-205.

22. Clarke H, Bonin RP, Orser BA, Englesakis M, Wijeysundera $\mathrm{DN}$, Katz J. The prevention of chronic postsurgical pain using gabapentin and pregabalin: a combined systematic review and meta-analysis. Anesth Analg. 2012;115(2):428-42.

23. Clarke H, Pereira S, Kennedy D, Andrion J, Mitsakakis N, Gollish $\mathrm{J}$, et al. Adding gabapentin to a multimodal regimen does not reduce acute pain, opioid consumption or chronic pain after total hip arthroplasty. Acta Anaesthesiol Scand. 2009;53(8):1073-83.

24. Nikolajsen L, Finnerup NB, Kramp S, Vimtrup AS, Keller J, Jensen TS. A randomized study of the effects of gabapentin on postamputation pain. Anesthesiology. 2006;105(5):1008-15.

25. Sen H, Sizlan A, Yanarates O, Emirkadi H, Ozkan S, Dagli G, et al. A comparison of gabapentin and ketamine in acute and chronic pain after hysterectomy. Anesth Analg. 2009;109(5):1645-50.

26. Sen H, Sizlan A, Yanarates O, Senol MG, Inangil G, Sucullu I, et al. The effects of gabapentin on acute and chronic pain after inguinal herniorrhaphy. Eur J Anaesthesiol. 2009;26(9):772-6.

27. Amr YM, Yousef AA. Evaluation of efficacy of the perioperative administration of Venlafaxine or gabapentin on acute and chronic postmastectomy pain. Clin $J$ Pain. 2010;26(5):381-5.

28. Brogly N, Wattier JM, Andrieu G, Peres D, Robin E, Kipnis E, et al. Gabapentin attenuates late but not early postoperative pain after thyroidectomy with superficial cervical plexus block. Anesth Analg. 2008;107(5):1720-5.

29. Cheng JK, Chiou LC. Mechanisms of the antinociceptive action of gabapentin. J Pharmacol Sci. 2006;100(5):471-86.

30. Clarke HA, Katz J, McCartney CJ, Stratford P, Kennedy D, Page $\mathrm{MG}$, et al. Perioperative gabapentin reduces $24 \mathrm{~h}$ opioid consumption and improves in-hospital rehabilitation but not postdischarge outcomes after total knee arthroplasty with peripheral nerve block. Br J Anaesth. 2014;113(5):855-64.

31. Gianesello L, Pavoni V, Barboni E, Galeotti I, Nella A. Perioperative pregabalin for postoperative pain control and quality of life after major spinal surgery. J Neurosurg Anesthesiol. 2012;24(2):121-6.

32. Pesonen A, Suojaranta-Ylinen R, Hammaren E, Kontinen VK, Raivio P, Tarkkila P, et al. Pregabalin has an opioid-sparing effect in elderly patients after cardiac surgery: a randomized placebo-controlled trial. Br J Anaesth. 2011;106(6):873-81.

33. Buvanendran A, Kroin JS, Della Valle CJ, Kari M, Moric M, Tuman KJ. Perioperative oral pregabalin reduces chronic pain after total knee arthroplasty: a prospective, randomized, controlled trial. Anesth Analg. 2010;110(1):199-207.

34. Clarke H, Wijeysundera D, Bonin R, Orser B, Englesakis M, Katz J. Pregabalin effective for the prevention of chronic postsurgical pain: really? In reply. Anesth Analg. 2013;116(2):508-9.

35. Singla NK, Chelly JE, Lionberger DR, Gimbel J, Sanin L, Sporn J, et al. Pregabalin for the treatment of postoperative pain: results from three controlled trials using different surgical models. J Pain Res. 2014;23(8):9-20.

36. Samad TA, Sapirstein A, Woolf CJ. Prostanoids and pain: unraveling mechanisms and revealing therapeutic targets. Trends Mol Med. 2002;8(8):390-6.

37. Clarke H, Woodhouse LJ, Kennedy D, Stratford P, Katz J. Strategies aimed at preventing chronic post-surgical pain: comprehensive perioperative pain management after total joint replacement surgery. Physiother Can. 2011;63(3):289-304 (Summer).

38. Koppert W, Wehrfritz A, Korber N, Sittl R, Albrecht S, Schuttler J, et al. The cyclooxygenase isozyme inhibitors parecoxib and paracetamol reduce central hyperalgesia in humans. Pain. 2004;108(1-2):148-53.

39. Sycha T, Anzenhofer S, Lehr S, Schmetterer L, Chizh B, Eichler $\mathrm{HG}$, et al. Rofecoxib attenuates both primary and secondary inflammatory hyperalgesia: a randomized, double blinded, placebo controlled crossover trial in the UV-B pain model. Pain. 2005;113(3):316-22.

40. Buvanendran A, Kroin JS, Tuman KJ, Lubenow TR, Elmofty D, Moric M, et al. Effects of perioperative administration of a selective cyclooxygenase 2 inhibitor on pain management and recovery of function after knee replacement: a randomized controlled trial. JAMA. 2003;290(18):2411-8.

41. Meunier A, Aspenberg P, Good L. Celecoxib does not appear to affect prosthesis fixation in total knee replacement: a randomized study using radiostereometry in 50 patients. Acta Orthop. 2009;80(1):46-50.

42. Fransen M, Anderson C, Douglas J, MacMahon S, Neal B, Norton R, et al. Safety and efficacy of routine postoperative ibuprofen for pain and disability related to ectopic bone formation after hip replacement surgery (HIPAID): randomised controlled trial. BMJ. 2006;333(7567):519.

43. Lakdja F, Dixmerias F, Bussieres E, Fonrouge JM, Lobera A. Preventive analgesic effect of intraoperative administration of ibuprofen-arginine on postmastectomy pain syndrome. Bull Cancer. 1997;84(3):259-63.

44. Romundstad L, Breivik H, Roald H, Skolleborg K, Romundstad PR, Stubhaug A. Chronic pain and sensory changes after augmentation mammoplasty: long term effects of preincisional administration of methylprednisolone. Pain. 2006;124(1-2):92-9.

45. Woolf CJ, Chong MS. Preemptive analgesia-treating postoperative pain by preventing the establishment of central sensitization. Anesth Analg. 1993;77(2):362-79.

46. Kissin I, Bright CA, Bradley EL Jr. The effect of ketamine on opioid-induced acute tolerance: can it explain reduction of opioid consumption with ketamine-opioid analgesic combinations? Anesth Analg. 2000;91(6):1483-8.

47. Perrin SB, Purcell AN. Intraoperative ketamine may influence persistent pain following knee arthroplasty under combined general and spinal anaesthesia: a pilot study. Anaesth Intensive Care. 2009;37(2):248-53.

48. Duale C, Sibaud F, Guastella V, Vallet L, Gimbert YA, Taheri $\mathrm{H}$, et al. Perioperative ketamine does not prevent chronic pain after thoracotomy. Eur J Pain. 2009;13(5):497-505.

49. Crousier M, Cognet V, Khaled M, Gueugniaud PY, Piriou V. Effect of ketamine on prevention of postmastectomy chronic pain. A pilot study. Ann Fr Anesth Reanim. 2008;27(12):987-93.

50. Suzuki M, Haraguti S, Sugimoto K, Kikutani T, Shimada Y, Sakamoto A. Low-dose intravenous ketamine potentiates epidural analgesia after thoracotomy. Anesthesiology. 2006;105(1):111-9.

51. Remerand F, Le Tendre C, Baud A, Couvret C, Pourrat X, Favard L, et al. The early and delayed analgesic effects of ketamine after total hip arthroplasty: a prospective, randomized, controlled, double-blind study. Anesth Analg. 2009;109(6):1963-71.

52. Sveticic G, Farzanegan F, Zmoos P, Zmoos S, Eichenberger U, Curatolo M. Is the combination of morphine with ketamine better than morphine alone for postoperative intravenous patient-controlled analgesia? Anesth Analg. 2008;106(1):287-93 (table of contents). 
53. Hayes C, Armstrong-Brown A, Burstal R. Perioperative intravenous ketamine infusion for the prevention of persistent postamputation pain: a randomized, controlled trial. Anaesth Intensive Care. 2004;32(3):330-8.

54. Katz J, Schmid R, Snijdelaar DG, Coderre TJ, McCartney CJ, Wowk A. Pre-emptive analgesia using intravenous fentanyl plus low-dose ketamine for radical prostatectomy under general anesthesia does not produce short-term or long-term reductions in pain or analgesic use. Pain. 2004;110(3):707-18.

55. Dullenkopf A, Muller R, Dillmann F, Wiedemeier P, Hegi TR, Gautschi S. An intraoperative pre-incision single dose of intravenous ketamine does not have an effect on postoperative analgesic requirements under clinical conditions. Anaesth Intensive Care. 2009;37(5):753-7.

56. Elia N, Tramer MR. Ketamine and postoperative pain: a quantitative systematic review of randomised trials. Pain. 2005;113(1-2):61-70.

57. Schley M, Topfner S, Wiech K, Schaller HE, Konrad CJ, Schmelz M, et al. Continuous brachial plexus blockade in combination with the NMDA receptor antagonist memantine prevents phantom pain in acute traumatic upper limb amputees. Eur $\mathbf{J}$ Pain. 2007;11(3):299-308.

58. Eisenberg E, Pud D, Koltun L, Loven D. Effect of early administration of the $N$-methyl-d-aspartate receptor antagonist amantadine on the development of postmastectomy pain syndrome: a prospective pilot study. J Pain. 2007;8(3):223-9.

59. Dahl JB, Moiniche S, Kehlet H. Wound infiltration with local anaesthetics for postoperative pain relief. Acta Anaesthesiol Scand. 1994;38(1):7-14.

60. Fassoulaki A, Sarantopoulos C, Melemeni A, Hogan Q. Regional block and mexiletine: the effect on pain after cancer breast surgery. Reg Anesth Pain Med. 2001;26(3):223-8.

61. Hollmann MW, Strumper D, Herroeder S, Durieux ME. Receptors, $\mathrm{G}$ proteins, and their interactions. Anesthesiology. 2005;103(5):1066-78.

62. Sugimoto M, Uchida I, Mashimo T. Local anaesthetics have different mechanisms and sites of action at the recombinant $N$ methyl-D-aspartate (NMDA) receptors. $\mathrm{Br} \quad \mathrm{J}$ Pharmacol. 2003;138(5):876-82.

63. Grigoras A, Lee P, Sattar F, Shorten G. Perioperative intravenous lidocaine decreases the incidence of persistent pain after breast surgery. Clin J Pain. 2012;28(7):567-72.

64. Fassoulaki A, Patris K, Sarantopoulos C, Hogan Q. The analgesic effect of gabapentin and mexiletine after breast surgery for cancer. Anesth Analg. 2002;95(4):985-91 (table of contents).

65. Karanicolas P, Cleary S, McHardy P, McCluskey S, Sawyer J, Ladak S, et al. Medial open transversus abdominis plane (MOTAP) catheters for analgesia following open liver resection: study protocol for a randomized controlled trial. Trials. 2014;15:241 (241-6215-15-241).

66. Taylor K, Rowbotham MC. Venlafaxine hydrochloride and chronic pain. West J Med. 1996;165(3):147-8.

67. Moulin D, Boulanger A, Clark AJ, Clarke H, Dao T, Finley GA, et al. Pharmacological management of chronic neuropathic pain: revised consensus statement from the Canadian Pain Society. Pain Res Manag. 2014;19(6):328-35.

68. Goldstein DJ, Lu Y, Detke MJ, Lee TC, Iyengar S. Duloxetine vs. placebo in patients with painful diabetic neuropathy. Pain. 2005;116(1-2):109-18

69. Yasuda H, Hotta N, Nakao K, Kasuga M, Kashiwagi A, Kawamori R. Superiority of duloxetine to placebo in improving diabetic neuropathic pain: results of a randomized controlled trial in Japan. J Diabetes Investig. 2011;2(2):132-9.

70. Raskin J, Pritchett YL, Wang F, D'Souza DN, Waninger AL, Iyengar S, et al. A double-blind, randomized multicenter trial comparing duloxetine with placebo in the management of diabetic peripheral neuropathic pain. Pain Med. 2005;6(5):346-56.

71. Wernicke JF, Pritchett YL, D'Souza DN, Waninger A, Tran P, Iyengar $\mathrm{S}$, et al. A randomized controlled trial of duloxetine in diabetic peripheral neuropathic pain. Neurology. 2006;67(8):1411-20.

72. Fassoulaki A, Triga A, Melemeni A, Sarantopoulos C. Multimodal analgesia with gabapentin and local anesthetics prevents acute and chronic pain after breast surgery for cancer. Anesth Analg. 2005;101(5):1427-32.

73. Lavand'homme P, De Kock M, Waterloos H. Intraoperative epidural analgesia combined with ketamine provides effective preventive analgesia in patients undergoing major digestive surgery. Anesthesiology. 2005;103(4):813-20.

74. Clarke H, Pereira S, Kennedy D, Gilron I, Katz J, Gollish J, et al. Gabapentin decreases morphine consumption and improves functional recovery following total knee arthroplasty. Pain Res Manag. 2009; 14(3):217-22.

75. Horlocker TT, Kopp SL, Pagnano MW, Hebl JR. Analgesia for total hip and knee arthroplasty: a multimodal pathway featuring peripheral nerve block. J Am Acad Orthop Surg. 2006;14(3):126-35.

76. Larson DW, Lovely JK, Cima RR, Dozois EJ, Chua H, Wolff $\mathrm{BG}$, et al. Outcomes after implementation of a multimodal standard care pathway for laparoscopic colorectal surgery. Br J Surg. 2014;101(8):1023-30.

77. Humble SR, Dalton AJ, Li L. A systematic review of therapeutic interventions to reduce acute and chronic post-surgical pain after amputation, thoracotomy or mastectomy. Eur J Pain. 2014.

78. Young EE, Lariviere WR, Belfer I. Genetic basis of pain variability: recent advances. J Med Genet. 2012;49(1):1-9.

79. Clarke H, Katz J, Flor H, Rietschel M, Diehl SR, Seltzer Z. Genetics of chronic post-surgical pain: a crucial step toward personal pain medicine. Can J Anaesth. 2014.

80. Sorge RE, Trang T, Dorfman R, Smith SB, Beggs S, Ritchie J, et al. Genetically determined $\mathrm{P} 2 \mathrm{X} 7$ receptor pore formation regulates variability in chronic pain sensitivity. Nat Med. 2012;18(4):595-9.

81. Nissenbaum J, Devor M, Seltzer Z, Gebauer M, Michaelis M, Tal M, et al. Susceptibility to chronic pain following nerve injury is genetically affected by CACNG2. Genome Res. 2010;20(9):1180-90.

82. Costigan M, Belfer I, Griffin RS, Dai F, Barrett LB, Coppola G, et al. Multiple chronic pain states are associated with a common amino acid-changing allele in KCNS1. Brain. 2010;133(9):2519-27.

83. Trescot AM. Genetics and implications in perioperative analgesia. Best Pract Res Clin Anaesthesiol. 2014;28(2):153-66.

84. Yang Z, Yang Z, Arheart KL, Morris R, Zhang Y, Rodriguez Y, et al. CYP2D6 poor metabolizer genotype and smoking predict severe postoperative pain in female patients on arrival to the recovery room. Pain Med. 2012;13(4):604-9.

85. Candiotti KA, Yang Z, Rodriguez Y, Crescimone A, Sanchez GC, Takacs P, et al. The impact of CYP2D6 genetic polymorphisms on postoperative morphine consumption. Pain Med. 2009;10(5):799-805.

86. Dahl JB, Kehlet H. Preventive analgesia. Curr Opin Anaesthesiol. 2011;24(3):331-8.

87. Katz J, McCartney CJ. Current status of preemptive analgesia. Curr Opin Anaesthesiol. 2002;15(4):435-41.

88. Katz J, Clarke H. Preventive analgesia and beyond: current status, evidence, and future directions. In: Rice A, Macintyre P, Walker M, Rowbotham D, editors. Clinical pain management. 2nd ed. London: Hodder Arnold; 2008.

89. Tumber PS. Optimizing perioperative analgesia for the complex pain patient: medical and interventional strategies. Can J Anaesth. 2014;61(2):131-40. 
90. Fisher RB, Johnson QL, Reeves-Viets JL. Pain management mini-series. Part II. Chronic opioid drug therapy: implications for perioperative anesthesia and pain management. Mo Med. 2013;110(3):231-5.

91. Cousins MJ, Power I, Smith G. 1996 Labat lecture: pain-a persistent problem. Reg Anesth Pain Med. 2000;25(1):6-21.

92. Ossipov MH, Dussor GO, Porreca F. Central modulation of pain. J Clin Invest. 2010;120(11):3779-87.

93. Shipton EA. The transition from acute to chronic post surgical pain. Anaesth Intensive Care. 2011;39(5):824-36.

94. Katz J, Asmundson GJ, McRae K, Halket E. Emotional numbing and pain intensity predict the development of pain disability up to one year after lateral thoracotomy. Eur J Pain. 2009;13(8):870-8.

95. Page MG, Katz J, Romero Escobar EM, Lutzky-Cohen N, Curtis $\mathrm{K}$, Fuss S, et al. Distinguishing problematic from non-problematic post-surgical pain: a pain trajectory analysis following total knee arthroplasty. Pain. 2015.

96. Burns LC, Ritvo SE, Ferguson MK, Clarke H, Seltzer Z, Katz J. Pain catastrophizing as a risk factor for chronic pain after total knee arthroplasty: a systematic review. J Pain Res. 2015;5(8):21-32.

97. Flor H, Fydrich T, Turk DC. Efficacy of multidisciplinary pain treatment centers: a meta-analytic review. Pain. 1992;49(2):221-30.
98. Morley S, Eccleston C, Williams A. Systematic review and meta-analysis of randomized controlled trials of cognitive behaviour therapy and behaviour therapy for chronic pain in adults, excluding headache. Pain. 1999;80(1-2):1-13.

99. Vowles KE, McCracken LM. Acceptance and values-based action in chronic pain: a study of treatment effectiveness and process. J Consult Clin Psychol. 2008;76(3):397-407.

100. Wetherell JL, Afari N, Rutledge T, Sorrell JT, Stoddard JA, Petkus AJ, et al. A randomized, controlled trial of acceptance and commitment therapy and cognitive-behavioral therapy for chronic pain. Pain. 2011;152(9):2098-107.

101. Fernandez MD, Luciano C, Valdivia-Salas S. Impact of acceptance-based nursing intervention on postsurgical recovery: preliminary findings. Span J Psychol. 2012;15(3):1361-70.

102. Lin LY, Wang RH. Abdominal surgery, pain and anxiety: preoperative nursing intervention. J Adv Nurs. 2005;51(3):252-60.

103. Wicksell RK, Olsson GL. Predicting and preventing chronic postsurgical pain and disability. Anesthesiology. 2010;113(6):1260-1.

104. Dworkin RH, Turk DC, Farrar JT, Haythornthwaite JA, Jensen MP, Katz NP, et al. Core outcome measures for chronic pain clinical trials: IMMPACT recommendations. Pain. 2005;113(1-2):9-19. 